\title{
A DECISION AID SYSTEM FOR HYDRAULIC POWER STATION REFURBISHMENT PROCEDURE
}

\author{
Francis and Kaplan Turbine
}

\author{
BELLET L., PARKINSON E.* AND AVELLAN F. \\ IMHEF $/ L M H / E P F L-A v$. de Cour,33 \\ 1007 LAUSANNE - Switzerland \\ AND \\ COUSOT T. AND LAPERROUSAZ E. \\ EDF-CNEH - Savoie Technolac \\ 73373 LE BOURGET DU LAC - France
}

\section{Project}

Maintenance policies for hydrogenerating equipment vary considerably from one operator to another, from a least cost strategy to the greatest care and tightest scheduling. However, whatever policy is adopted, sooner or later maintenance is no longer enough to uphold acceptable levels of performance or safety. When that happens, renovation or refurbishment takes over.

In general, the decision to refurbish a scheme is inspired by a combination of causes, which can be grouped in three types: age, downgraded performance, and unsuitable or costly operating techniques.

It is fairly difficult to evaluate the effect of age, although it is well known that, as time passes, the risk of a serious accident increases. Obviously, refurbishment consecutive to an accident cannot be scheduled and can therefore not take place at the same cost nor with the same results as a scheduled refurbishment that is correctly integrated into the generating program.

Even the most painstaking maintenance works are not enough to preserve initial performance levels. Energy losses increase inexorably everywhere in the system: hydraulic, mechanic and electric losses.

Finally, operation becomes increasingly difficult. The original design requirements, such as operating limits imposed by cavitation or instability, are compounded by constraints due to wear and permanent deformations (watertightness of guide vanes or gates and valves) and the safety of the plant in general. Operating losses due to the frequency of maintenance must also be considered, and even, within a cascade of plants, the unsuitability of one scheme versus those upstream and downstream from it. 
Furthermore, although the raison d'être of power plants remains the generation of energy at least cost, other needs or requirements have grown up over the years such as:

- the safety of facilities;

- the match between performance levels and network requirements;

- compliance with new legislation;

- new operating requirements (irrigation, low stage support, recreational uses).

A well-managed refurbishment must take all these new data into account, as well as technological and scientific advances. Depending on those new requirements, a refurbishment operation can take many forms, from replacement of elements with identical equipment to the construction of a whole new plant. Compromises also exist, such as replacing a runner with one of modern design. In general the objective is to minimise the cost of the operation or guarantee that it is cost-effective because of improved generating performances.

Improving performance by replacing the runner alone, and when necessary modifying the generator, means extra cost and risk in comparison to replacement with identical equipment, extra cost because more study is required (and even model tests), and risk because it can never be absolutely guaranteed that performance will actually improve.

The IMHEF and EDF have been working together for several years on various subjects relating to hydraulic machinery. Since 1995, the two have joined forces to develop a procedure for the evaluation of older turbines. This joint work was inspired by the realisation of two facts:

1- EDF is confronted with an ageing stock of hydro facilities and the renew al of many of its concession agreements. EDF's engineers working in hydro generation are reflecting on how to enhance the value of French facilities, as part of a project called "Hydraulique Demain" ("The Hydraulics of Tomorrow"). This project is aimed at implementing the technico-economic tools and methods required to rationalise expenditures and to analyse the potential of hydro facilities.

2- The IMHEF and EDF have long worked for industrial groups, operators or water resources managers as consulting engineers. With the resulting awareness of those entities' needs in refurbishment projects, they have decided to adapt modern techniques to that field.

Our work is part of the global process required to prepare for a refurbishment, which among other things includes technical, operating and economic studies. Our objective is to ascertain how suitable a machine is for its site and to evaluate the energy potential of the stationary elements of an existing turbine. We therefore first focus on those stationary parts (spiral casing, distributor and where necessary headrace pipes) and then on the runner if necessary.

Such a study can be scheduled in various stages of a project, and can have various goals. When it is scheduled before design, it is a decision- 
making tool that helps in establishing the technical and economic balance sheet on a facility and in orienting the choice of what type of refurbishment to plan. If the decision is made to change the runner, it will serve in setting up the technical specifications for the new runner on solid bases and when necessary in predicting whether changes will be needed to stationary parts.

\section{Refurbishment procedure}

There are a multitude of components in a hydroelectric scheme, and therefore the engineer in charge of study of a refurbishment is faced with a wide and complex range of options. To guide his choices, he must take into account both technical and economic aspects, without neglecting legal aspects (the concession agreement) and environmental aspects, which may also play a decisive role. The procedure described herein concerns only technical aspects and is intended to help in establishing a rapid and accurate balance sheet on the site under study in order to be able to conduct an economic study. This procedure is divided into three stages, progressing from a global approach to a specific approach.

\subsection{PHASE 1:RENOVA}

The first stage in a refurbishment study consists in gathering all the available information on the project at hand concerning the hydraulic elements in the scheme, with the purpose of drawing up a balance sheet on the following main elements: water intakes, penstocks, gates and valves, turbine, headrace and tailrace channels. The information collected is used to identify critical zones in the scheme that the study will focus on. A complete and systematic summary can be transcribed in computer files of characteristics to create a data base, called Renova. This data base was created as part of the present project in order to:

- inventory existing machines described in the literature, whether refurbished or not;

- run a statistical study based on those machines;

- establish a data base for refurbishment projects.

In the statistical study, the machine at hand is compared to other machines, whether modern or older, and where appropriate a new type of turbine can be defined that is better suited to the site's present characteristics. This data base is for the moment essentially concentrated on the turbine, but can easily be extended to the other elements in a scheme.

\subsection{PHASE 2:RENOVATURB}

The second phase is more specific, as it concerns the high pressure parts of the turbine, i.e., the spiral casing and distributor. Its objective is to precisely determine conditions upstream from the runner. The Renovaturb 
computer program, which was partially developed during this project, allows geometrical analysis of those components:

- verification and computation of geometrical quantities (sections laws, skeletons lines, distributor opening law, opening, etc.);

- prediction of flow angles at the spiral casing outlet;

- compatibility between the estimated angles and actual angles of stay vanes;

- compatibility of geometrical angles between stay vanes and guide vanes;

- automatic generation of a structured mesh of the distributor and semiautomatic of the spiral casing in preparation for phase 3 .

\subsection{PHASE 3:COMPUTER MODELLING}

Knowledge of hydraulic conditions in the high pressure parts of the turbine (spiral casing and distributor) is very important, and a computer model allows a more in-depth analysis of each component. Phase 2 of this procedure gives an estimation of the average flow angle, defined as $\alpha=\operatorname{atan}\left[\frac{C_{m}}{C_{u}}\right]$ where $C_{m}$ and $C_{u}$ are respectively the meridional and tangential velocity, at the spiral casing outlet. If a more precise idea of the phenomena in the spiral casing is desired, a numerical calculation can be done, although it must be borne in mind that considerable time is needed for calculation and generation of the mesh (as the tong is specific to each spiral casing, generation of it must be modified in the Renovaturb program).

Calculation of the distributor gives the changes in flow angle as well as those in kinetic moment, so that the maximum energy available at the runner inlet and the flow angle are known. The runner computation will indicate whether it is capable of transforming this available energy. The geometry of the blades and the meridian channel are defined on the basis of drawings or from site measurements (articulated arm or theodolite) if no drawings are available or if the runner's geometry has been changed.

A study of the draft tube has not been envisaged for the moment, although it is a major element in the turbine, because of the complexity of the unsteady phenomena that take place in it. Calculation of this element with a Navier-Stokes program has still not been validated experimentally, and so cannot be systematically employed. Two types of computation have been envisaged for this procedure:

- Calculation using a Euler type code: the EULER-IMHEF [5]. The CALECHE (C) code (Metraflu), a finite element code based on a structured mesh, will be used for future calculation of the runner;

- Calculation with a Navier-Stokes type code: the N3S (C) [1] code (EDFSimulog) in its turbomachine version. 


\section{Application to a real-life case}

The Pinet hydropower plant on the Tarn river in France was chosen to validate this procedure. It was commissioned in 1929, with five vertical axis Francis turbines, each outputting maximum mechanical power of $8 \mathrm{MW}$ at $250 \mathrm{rpm}^{-1}$ for a net head of $32 \mathrm{~m}$ and a discharge of $Q=30.4 \mathrm{~m}^{3} / \mathrm{s}$. The Thoma number $\sigma$, or cavitation factor, defined as being the ratio between net positive suction specific energy and specific energy is close to 0.3 .

\subsection{PHASE 1}

Data gathered on the site, including some of the original drawings, were used in this study. A statistical comparison of the existing turbine with the Francis turbines inventoried in the data base is presented in Figures 1 and 2. The dimensionless discharge and energy coefficients are defined as $\varphi=\frac{Q}{\pi \cdot \varphi_{i} \cdot R^{3}}$ and $\psi=\frac{2 \cdot E}{\omega^{2} \cdot R^{2}}$, where $\omega$ is the rotating speed and $R$ the raduis. The following observations can be made:

- Specific speed, defined as $\nu=\frac{\omega \cdot \sqrt{\frac{Q}{\pi}}}{(2 . E)^{3 / 4}}$, which is representative of the type of turbine used, is well suited to the available head.

- The reference runner diameter, representative of the turbine's characteristic dimensions, is well suited to the discharge through the turbine.

- The reference diameter, on the other hand, is not suited to the mechanical power output by the runner. It is therefore possible, with a better runner, to supply more mechanical power to the shaft.

- The coefficient $\varphi^{2} / \psi$, that is representative of the specific kinetic energy at the runner outlet versus head, is within the statistical average. This coefficient is also proportional to energy losses in the draft tube, and therefore would seem to be suited to the runner being used.

- The Thoma number $\sigma$, or cavitation factor, is not isolated from the other values for Francis turbines (the number of values is reduced, since $\sigma$ is not routinely given in the literature). However, this factor alone cannot give information on turbine elevation setting, which is why the coefficient $\kappa$, defined as $\kappa=\frac{\psi_{\overline{1}}}{\operatorname{tg} \beta_{\overline{1}}} \cdot \sigma_{\min }$ where $\beta_{\overline{1}}$ is the relative flow angle at the runner trailing edge, has been calculated.

- The lower turbine setting acceptable for this machine requires that coefficient $\kappa \simeq 1$. A considerable margin of safety was taken by using a value of 3.5 .

On the basis of these observations, it was proposed that a Francis runner with a smaller diameter would be better suited to the present operating conditions. A statistical comparison with the Kaplan turbines in the inventory (figure 1) shows that the use of a Kaplan (or Impeller) turbine with this reference diameter could also be envisaged.

It should be noted that almost none of the original equipment has been replaced (generators, transformers, runner, etc.), and, as there is no reg- 

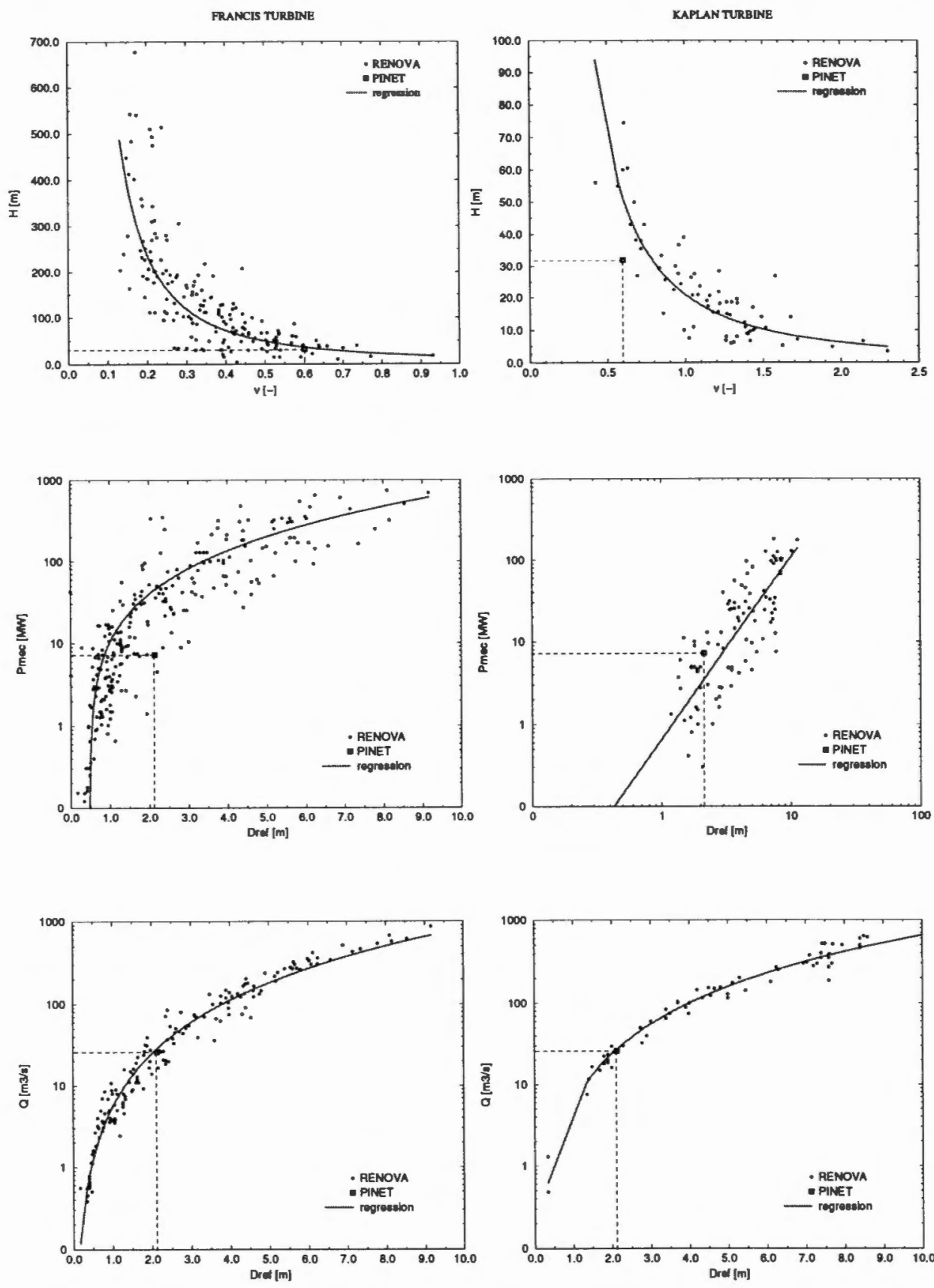

Figure 1. Head/Specific speed - Mechanical power/Diameter - Discharge/Diameter 

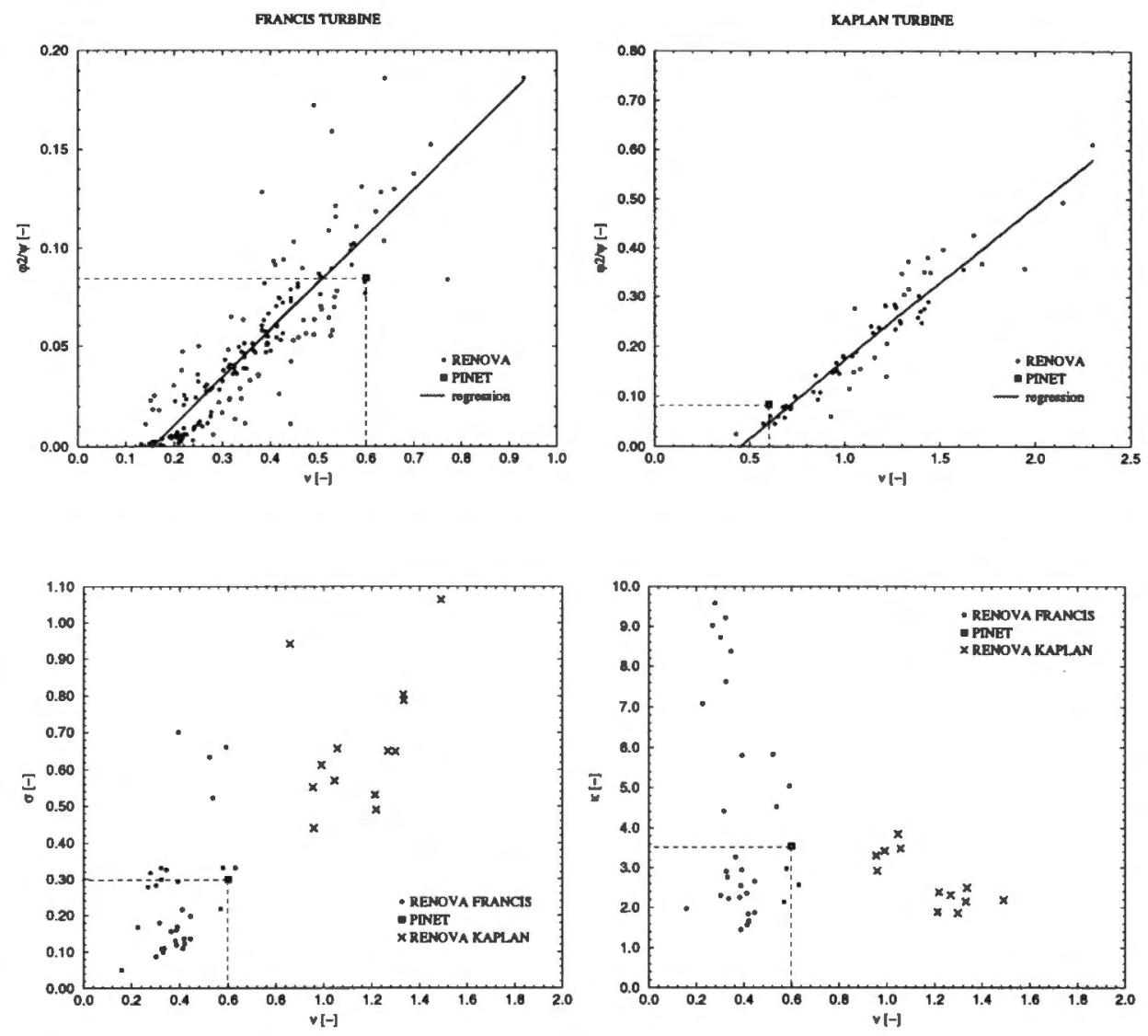

Figure 2. Coefficient $\frac{\varphi^{2}}{\psi} /$ Specific speed - Thoma number/Specific speed - Coefficient $\kappa /$ Specific speed

ulating device, the turbines always run at the same operating point. The essential information gathered on the turbine was the following:

- Major losses were measured in the inlet valves, as well as in the fivebranch manifold upstream from those valves.

- The double curve spiral casing has two by-passes that divert flow directly downstream from the turbine in the event of any problem, and leakage has been observed at the outlet from those elements.

- The runner is suffering from relatively major erosion due to cavitation at the pressure-side inlet of the blades near the runner band. 


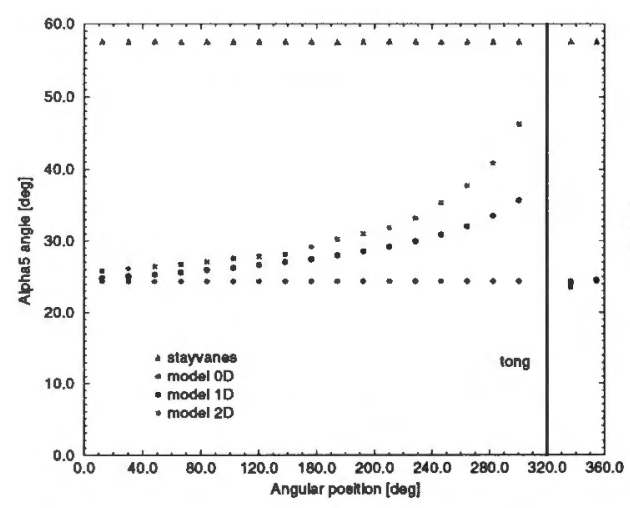

Figure 3. Estimated angles at the spiral casing outlet

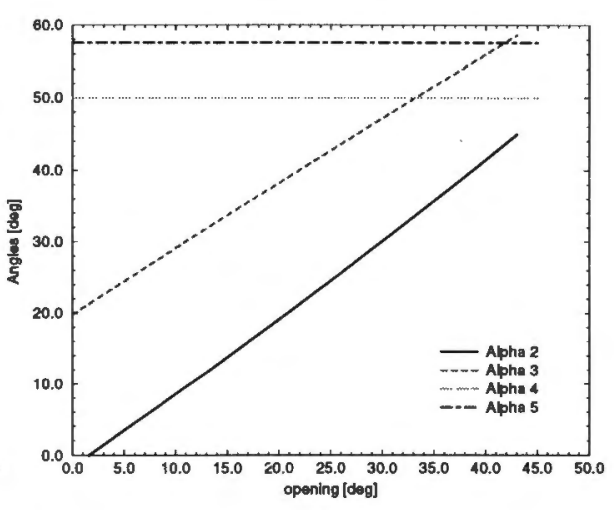

Figure 4. Geometrical angles in the distributor

\subsection{PHASE 2}

Index $5 \& 4$ and $3 \& 2$ are corresponding respectively to the stay vane leading \& trailing edge and guide vane leading \& trailing edge. Three models for evaluation of average hydraulic flow angle at the spiral casing outlet have been programmed into Renovaturb and are shown in figure 3 . The assumptions applied are the following:

- model 0D: $C_{u}=$ cste at the inlet and $C_{r}=$ cste at the outlet.

- model 1D: discharge is absorbed evenly around the machine.

- model 2D: the spiral casing is considered as a coiled pipe where headloss is assumed constant per unit of length.

It can be noted that the skeleton angle at the leading edge of the stay vanes is 57.6 [deg] while the models used give an average angle close to 28 [deg]. We may therefore expect major incidence losses at this location. On Figure 4, we note an intersection of the curves representing angles $\alpha_{3}$ and $\alpha_{4}$ for an opening of 33.18 [deg], where losses would be at a minimum in the distributor. As the opening used was 33.5 [deg], the setting of the stay vane is well suited to the setting of the guide vane.

\subsection{PHASE 3}

The distributor is of the radial type with adjustable guide vanes. It is composed of symmetrical stay vanes and guide vanes. For the Euler calculation, a structured mesh of a channel between blades, shown in Figure 5, was created with 29,600 nodes: $I_{\max }=74 \quad J_{\max }=20 \quad K_{\max }=20$, where direction $i$ corresponds to the main direction of flow, and $k$ to the height of the distributor. 10,000 iterations, i.e. one hour of computation time on an IBM Risc 6000-3AT work station $(196 \mathrm{Mb})$ were required. The mesh for the 
N3S computation was obtained from a structured hexahedral mesh measuring $70 \times 16 \times 15$ divided into tetrahedrons to give an unstructured mesh of 125,941 nodes. The computation was done on EDF's Research and Development Department's Cray X-MP, with 1000 iterations and 3.5 hours of computation time.

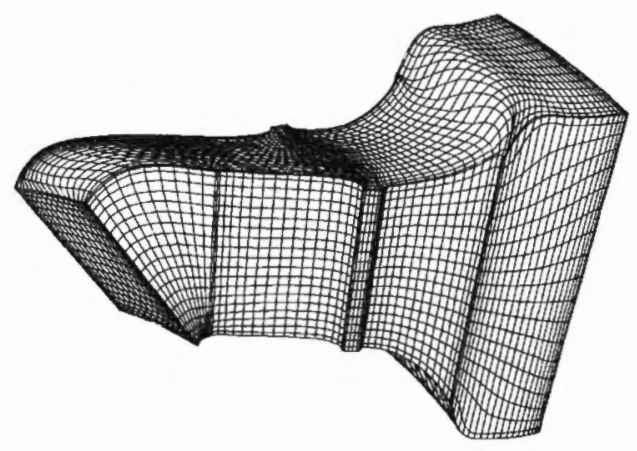

Figure 5. Structured mesh of the computational domain

\subsubsection{Results of the Euler computation}

For each side of the mesh $i$ the average value of kinetic moment and hydraulic flow angle $\alpha$ was calculated (Figure 6), using the following formula:

$$
\overline{r . c_{u}}=\frac{\int_{A_{i}} r c_{u} \vec{c} \cdot \vec{n} d A}{\int_{A_{i}}} \quad \overline{\vec{c} \cdot \vec{n} d A} \quad \overline{\operatorname{tg} \alpha_{i}}=\frac{\overline{r \cdot c_{m}}}{\overline{r . c_{u}}}=\frac{\int_{A_{i}} r c_{m} \vec{c} \cdot \vec{n} d A}{\int_{A_{i}} r c_{u} \vec{c} \cdot \vec{n} d A}
$$

Two calculations, one with an initial angle of 28 [deg] and the other with 24.37 [deg], were run. Despite the difference in the flow angle at the distributor inlet, the average kinetic moment and the average flow angle at the outlet remained unchanged. It was observed that the average flow angle at the distributor outlet $\alpha_{2}$ is about 37.25 [deg], while the geometric angle of the camber line $\alpha_{2 g e o m}$ is 34 [deg] (see Figure 4). Maximum dimensionless energy available at the runner inlet, assuming that we are close to the top point and that $U . C_{u} \simeq 0$, is $\psi_{d i s p}=0.628\left(2 . r . c_{u}=0.7\right)$.

\subsubsection{Results of the Navier-Stokes computation}

For the same flow inlet conditions, the average flow angle at the distributor outlet is about 33.0 [deg], so 4 [deg] lesser than the Euler computation. The viscosity effect on the outlet flow angle is significant. Maximum dimensionless energy available at the runner inlet is $\psi_{d i s p}=0.73$. Since the net dimensionless energy available for the turbine is $\psi_{\overline{1 e}}=0.806$ and the efficiency (measured) is close to $87 \%$, the dimensionless transformed energy is about $\psi_{m e c}=0.701$, which is in accordance with the computational $\psi_{d i s p}$ value. 


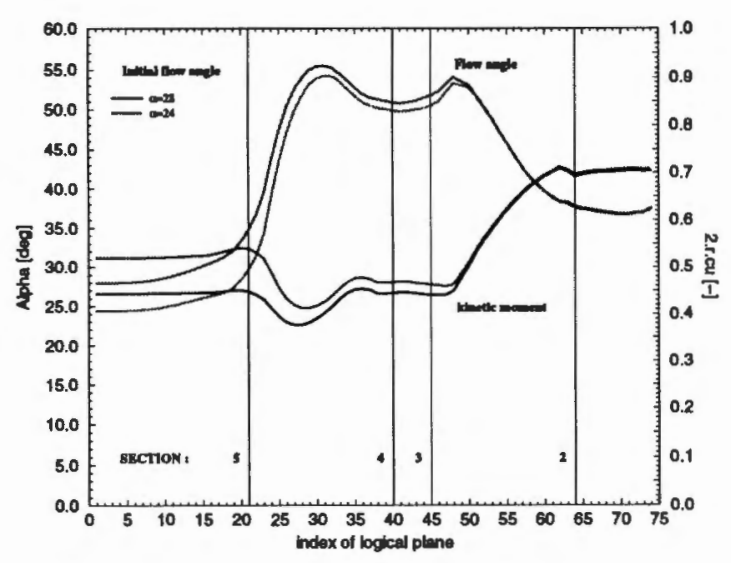

Figure 6. Flow angle and kinetic moment

\section{4. conclusion}

This first study was the opportunity to set up an overall balance sheet on the hydraulic elements of the scheme and to emphasis sensitive points. The next stages in the project will be to measure runner geometry on site, in order to analyse it and to have all the elements needed to define, from a technical standpoint, the modifications required.

\section{Acknowledgments}

The authors wish to thank J-F Combes and E. Dueymes of EDF-DER for their help in achieving the N3S computations. They wish, also, thank Hydro-Vevey S.A and Sulzer Hydro for their contribution of the development of Renovaturb.

\section{References}

1. J.-P. Chabard, B. Metivet, G. Pot, and B. Thomas. An efficient finite element method for the computation of $3 \mathrm{~d}$ turbulent incompressible flows. Finite Elements in Fluids, vol. 8, 1992.

2. EPRI. Hydropower Plant Modernization Guide-Hydroplant modernization, volume 1. Electric Power Research Institute, 1989.

3. EPRI. Hydropower Plant Modernization Guide-Turbine Runner Upgrading, volume 2. Electric Power Research. Institute, 1989.

4. P. Henry. Turbomachines hydrauliques. Presses polytechniques et universitaires romandes, 1992.

5. E. Parkinson, P. Dupont, R. Hirschi, J. Huang, and F. Avellan. Comparison of flow computation results with experimental flow surveys in a Francis turbine. In XVII IAHR International Symposium, Beijing, China, September 1994.

$\left({ }^{*}\right)$ Now at Hydro-Vevey 